\title{
Larval Metal Pollutant Exposure Alters the Epigenetic Architecture of the Major Malaria Vector Anopheles Arabiensis (Diptera: Culicidae)
}

\section{Alexander C.S.N. Jeanrenaud}

University of the Witwatersrand

Basil D. Brooke

University of the Witwatersrand

Shune Oliver ( $\nabla$ shuneo@nicd.ac.za )

National Institute for Communicable Diseases https://orcid.org/0000-0003-0658-6658

\section{Research}

Keywords: Anopheles arabiensis, Epigenetic, phenotypic, mosquitoes

Posted Date: July 30th, 2020

DOI: https://doi.org/10.21203/rs.3.rs-49568/v1

License: (c) (i) This work is licensed under a Creative Commons Attribution 4.0 International License.

Read Full License 


\section{Abstract}

\section{Background}

Anopheles arabiensis (a member of the An. gambiae species complex) is a major vector of malaria. These mosquitoes typically breed in clean bodies of water, but can also utilise polluted waters. Although this expands their breeding range, adaptation to breeding in polluted waters necessitates coping with a large amount of environmental stress in the form of exposure to heavy metal pollutants. Environmental stress can induce heritable changes to an organism without altering the basal genetic code. This occurs by rapid changes in the regulatory elements associated with nucleic acids that result in changes in patterns of gene expression and silencing. This is known as epigenetic regulation. There is currently little information on epigenetic regulation in An. arabiensis. The aim of this study was to examine whether exposure to metal pollutants at the larval stage induced alterations of epigenetic markers resulting in phenotypic changes in insecticide susceptible and resistant laboratory strains of An. arabiensis.

\section{Results}

There was a marked difference in the phenotypic response in adult mosquitoes of the insecticide susceptible strain compared to that of its' resistant counterpart. The susceptible strain showed reduced fertility and fecundity in response to epigenetic manipulation. For males and females of the resistant strain, exposure to nucleic acid modifying drugs typically increased their tolerance to insecticides. In the insecticide susceptible strain, regulatory signals associated with gene silencing were increased, while those associated with gene expression were reduced in response to heavy metal exposure. By contrast, the insecticide resistant strain showed a decrease in signatures associated with gene silencing as well as an increase in signatures associate with chromatin remodelling.

\section{Conclusions}

Larval exposure to heavy metal contaminants can result in heritable changes in gene expression. These data suggest that exposure to the same pollutants can result in different biological outcomes in insecticide susceptible and resistant adult An. arabiensis, and that these changes are reflected in their respective global? epigenetic signatures.

\section{Introduction}

The DNA methylation that occurs in invertebrates is essentially identical to that of mammals although there is greater interspecific variation in DNA methylation percentages in insects [1]. In contrast to mammals, DNA methylation in insects and plants is restricted to transcribed gene regions [2-4]. Widespread DNA methylation patterns are found across insect orders, namely: Diptera, Lepidoptera, Coleoptera, Hemiptera, and Blattodea, with high amounts of diversity [5]. Interestingly, the Leipdoptera, Coleoptera and Hymenoptera all show reduced levels of DNA methylation when compared to other orders, 
but none as significantly low as Diptera. It is believed that the ability to methylate DNA at high levels was lost early in Dipteran evolution $[5,6]$.

Although present in very low amounts, methylated DNA residues are present within Dipteran insects, specifically Anopheles gambiae and several Drosophila spp [7].. A study examining methylation in Diptera looked primarily at 5-methyl cysteine $(5-\mathrm{mC})$ methylation and not 5-hydoxymethyl cysteine (5-hmC) methylation [7].

As gene expression can be regulated by various factors including heterochromatin proximity and RNA modifications, various studies have examined the role of histone and RNA modifications in invertebrates [8]. Currently, it appears that histone modifications, specifically methylation, are closely related to changes in DNA methylation. This has been observed in a number of insect species including Solenopsis invicta (red imported fire ant) and Camponotus floridanus (Florida carpenter ant), and across insect orders [9-12]. In Drosophila, genome-wide epigenomic profiles of histone modifications have been established, with this information being correlated to functional domains and regulatory elements $[13$, 14]. In mosquitoes, however, almost no data of this calibre is available, with few studies examining these post-translational modifications [15]. It has been established that histone methyltransferases are present within mosquitoes, specifically anophelines, and that methylation may inhibit transcription with acetylation promoting it [15]. There is little if any information regarding histone or RNA modifications in Anopheles mosquitoes, particularly An. arabiensis.

Epigenetics plays a significant role in the expression of genes during periods of environmental stress [16, 17]. Therefore, examining how epigenetic architectures change in mosquitoes following pollutant exposure is important. Moreover, very few studies have examined the effects of epigenetic alterations on mosquitoes from a phenotypic perspective. Relevant studies to date include the epigenetic effects on cold-hardiness and insecticide resistance in Aedes aegypti $[18,19]$. In these studies, it was shown that epigenetic alterations allow for short-lived transgenerational effects in terms of cold-hardiness of eggs as well as altering the insecticide sensitivities in Aedes. In malaria vectors, it follows that epigenetic alterations may have the capacity to alter life history traits of epidemiological importance, including vectorial capacity and adaptability to xenobiotics and, as such, it is important to understand whether larval pollutant exposure can alter adult epigenetic patterns.

Anopheles arabiensis is a major malaria vector species within the An. gambiae species complex [20] and is one of the vectors responsible for the transmission of malaria in South Africa [21]. This species is difficult to control due to its' propensity to bite and rest outdoors, making it less susceptible to traditional control methods that target indoor-biting and -resting females [22]. Furthermore, the outdoor-biting behaviour of $A n$. arabiensis means that it can sustain low-level transmission in a control setting, causing residual malaria [23], which threatens South Africa's malaria elimination agenda.

In terms of adaptive characteristics, insecticide resistance is widespread in An. arabiensis [24]. There are also reports of populations of this species adapting to breeding in polluted water $[25,26]$, as opposed to the clean, sunlit temporary bodies of water that are typically chosen by member species of the An. 
gambiae complex [27]. This adaptive characteristic has been associated with increased insecticide tolerance [28-30], as well as a number of other factors that alter life history [31, 32].

Patterns of insecticide resistance and other adaptations of epidemiological relevance that respond to environmental stressors may be associated with epigenetic systems in mosquitoes. The aim of this study was therefore to examine whether exposure to metal pollutants at the larval stage induces alterations of epigenetic markers resulting in phenotypic changes in insecticide susceptible and resistant laboratory strains of An. arabiensis.

\section{Materials}

Two An. arabiensis strains were used in this study. The SENN strain was colonised from Sennar, Sudan in 1980. From this strain SENN-DDT, an insecticide resistant strain, was selected. SENN-DDT has been continuously selected for DDT resistance since 1995. SENN is insecticide susceptible, while SENN-DDT is resistant to DDT, permethrin, deltamethrin, $\lambda$-cyhalothrin and malathion $[33,34]$. Resistance in this strain is mediated by a combination of the L1014F mutation and elevated cytochrome P450, GST and general esterase activity [35]. Pyrethroid resistance in SENN-DDT is of low intensity [36]. Both strains are housed in the Botha de Meillon insectary of the National Institute for Communicable Diseases (NICD), Sandringham, Johannesburg. Mosquitoes were reared at $25^{\circ} \mathrm{C}\left( \pm 2^{\circ} \mathrm{C}\right)$ and at $85 \%$ humidity $( \pm 5 \%)$ with a 12:12 hr light/dark cycle. Larvae were fed a combination of yeast and dog biscuits [37].

\section{Methods}

\section{Summary of}

\section{methods}

This study is divided into two sections. The first examined the interplay between epigenetics and phenotype. This was done by examining the effects of known epigenetic modulators on fertility and fecundity as well as examining their effect on insecticide resistance phenotype. The effect of metal exposure on these phenotypes in these particular strains has been characterised previously $[29,38]$.

The second portion of the study focussed on epigenetic architecture. In this study, the effect of epigenetic modulators on DNA methylation associated with gene expression was quantified. For the remaining experiments, the effect of larval metal pollution on the epigenetic architecture of the subsequent adults was characterised. This was done by examining the effects of RNA m6A methylation, 5-mC and 5-hmC DNA methylation as well as Histone Acetyl-transferase (HAT) activity.

Characterisation of the effects of epigenetic modulators on An. arabiensis phenotype

The effect of epigenetic modulators on sex-linked oviposition and larval hatch percentage 
SENN and SENN-DDT L1 larvae were reared in clean water. Approximately 200 emerged adults (mixed sex; <24 hours) were exposed to $5 \mu \mathrm{M}$ DZNep, $5 \mu \mathrm{M}$ genistein or $5 \mu \mathrm{m}$ vinclozolin, separately $[19,39]$. DZNep is a known histone methylation inhibitor [40]. The phytoestrogen genistein modulates chromatin and reduces hypermethylation [41]. The fungicide vinclozolin has been shown to be capable of inducing transgenerational changes [42]. These compounds were administered via a $10 \%$ sucrose solution. Control samples were provided with untreated sucrose water. Adults were exposed to treatments for three days, after which time they were separated into cages at a ratio of 40 males to 20 females. Adults were provided one blood meal on day three (Ethics clearance number: University of the Witwatersrand S Oliver 03-01-2018) and allowed to oviposit two days post this meal. Bowls containing the eggs were kept for three days before being frozen. Eggs and hatched larvae were then enumerated by physical counting, and hatch percentage was calculated as (hatched larvae/total unhatched eggs + hatched larvae) x100.

The effect of genistein, vinclozolin and DZNep exposure on insecticide resistance phenotypes in the insecticide resistant SENN-DDT strain

SENN-DDT adults emerging from larvae reared in clean water were separated into cages ( 200 each; mixed sex) where they were exposed to genistein, vinclozolin, or DZNep in the concentrations described above. Each compound was administered via a $10 \%$ sucrose solution. Controls were provided with $10 \%$ untreated sucrose water. Following three days of exposure to treated sucrose water, adults were exposed to the insecticides deltamethrin, malathion, permethrin, DDT and $\lambda$-cyhalothrin for one hour using standard WHO bioassays [43]. Final adult mortalities were recorded $24 \mathrm{~h}$ post exposure. Females were not allowed access to blood meals for the duration of their lives. Epigenetic agent-treated adults constituted the experimental group, while untreated adults constituted the control group. For each exposure, an unexposed sample was used as an environmental control, and a sample exposed to the relevant insecticide solvent used as a general control (solvent control). If either of these control mortalities exceeded $10 \%$, the assay was discarded.

For all phenotypic assays, exposure to none of the sucrose administered epigenetic agents induced significant mortality.

\section{Characterisation of the epigenetic architecture of the SENN and SENN-DDT strains}

Culturing of pollutantexposed An. arabiensis

200 SENN or SENN-DDT larvae were reared per replicate in water polluted with $0.36 \mu \mathrm{g} / \mathrm{L}$ cadmium chloride, $1.86 \mu \mathrm{g} / \mathrm{L}$ copper nitrate or $4.39 \mu \mathrm{g} / \mathrm{L}$ lead nitrate. The control constituted of larvae reared in unpolluted water. These pollutants were selected due to their ubiquity in larval polluted water and because of their effects on the phenotype and biochemistry of the SENN and SENN-DDT strains as has been characterised $[29,44-46]$. The concentrations selected represent the legally accepted maximum acceptable toxic concentrations (MATC). These represent the maximum concentrations that these metals 
are allowed to be in water before it is considered polluted. These concentrations are therefore a proxy for the minimum amount of pollutant stress or selection pressure a body of water considered polluted can exert.

The exposed larvae were reared to adulthood in the Botha de Meillon insectary, Johannesburg as per [37]. Adults were allowed ad libitum access to $10 \%$ sucrose and females not allowed blood throughout their lifetime.

\section{Determination of global RNA methylation change in response to heavy metal exposure}

Global changes in RNA methylation were quantified by ELISA. The emerging adults' ( $<24$ hours) RNA was extracted using Quick-RNA ${ }^{\mathrm{TM}}$ Miniprep kit (Zymo Research: R1055) with 5 adults/tube. RNA integrity was assessed by $1 \%$ agarose $0.5 \mathrm{X}$ TBE gel at $70 \mathrm{~V} / 500 \mathrm{~mA}$ for 30 minutes (RiboRuler ${ }^{\mathrm{TM}}$ High Range RNA ladder (Fermentas: \#SM1823)). To ensure RNA integrity, samples were kept on ice when in use and stored at $-70^{\circ} \mathrm{C}$. Global RNA methylation was quantified using the m6A RNA Methylation Colorimetric Assay Kit $\left(\right.$ Abcam $^{\text {TM }}$ : ab185912). Sample colour changes were quantified using a Multiskan Ascent (Thermo ${ }^{\text {TM }}$ Electron Corporation) and Ascent Software version 2.6 at $405 \mathrm{~nm}$.

\section{Determination of global 5-mC and 5-hmC adult DNA methylation patterns}

DNA was extracted from newly emerged adults ( $<24$ hours) (5 adults/extraction tube; 12 extractions per set) using using the NucleoSpin ${ }^{\circledR}$ Macherey-Nage ${ }^{\text {TM }}{ }^{\text {kit }}$ (Catalogue number: 740470.50). Extracted DNA samples were then utilised in the Imprint ${ }^{\circledR}$ Methylated DNA Quantification Kit (Sigma Aldrich: MDQ196RXN) as per the manufacturer's protocol. 5-hmC quantification was performed using the Quest 5-hmC ${ }^{\mathrm{Tm}}$ DNA ELISA Kits (Zymo Research: D5426), as per the manufacturer's protocol. Sample colour changes were quantified using a Multiskan Ascent $\left(\right.$ Thermo $^{\mathrm{TM}}$ Electron Corporation) and Ascent Software version 2.6 at $450 \mathrm{~nm}$.

\section{Determination of Histone Acetyl Transferase activity}

Nuclear protein was extracted from adult mosquitoes in order to determine histone acetyl transferase (HAT) activity. In brief, SENN and SENN-DDT adults were collected upon emergence from water that was either untreated (control), or treated with cadmium chloride, copper nitrate or lead nitrate as with the nucleic acid methylation experiments. Newly emerged adults were cold-killed at $-70^{\circ} \mathrm{C}$. Five mosquitoes of each strain, sex and treatment were used per replicate. The mosquitoes were homogenised in $0.1 \mathrm{M}$ Phosphate Buffered Saline (PBS) pH 7.2 supplemented with protease inhibitors with a final concentration of $2 \mathrm{mM}$ leupeptin, $4 \mathrm{mM}$ PMSF and $5 \mathrm{mM}$ EDTA. Samples were homogenised in a Tissue Lyser II homogeniser (Qiagen) for 10 minutes at a frequency of $25 \mathrm{~Hz}$. Chitin proteins were separated by centrifugation at $500 \mathrm{xg}$ at $4{ }^{\circ} \mathrm{C}$ for 2 minutes. Cell pellets were collected after centrifugation at $500 \mathrm{xg}$ at 4 ${ }^{\circ} \mathrm{C}$ for 10 minutes. Nuclear protein was extracted from this packed cell volume using the NucBuster ${ }^{\text {TM }}$ Protein extraction kit (Novagen: 71183-3). Protein was quantified using the Braford method [47]. Fifty $\mu \mathrm{g}$ 
of protein was used as homogenate to determine HAT activity using a commercial HAT activity calorimetric activity kit (Sigma Aldrich: EPI001). Activity was monitored hourly for 5 hours at $440 \mathrm{~nm}$ using a Spectramax ABS plus 96-well plate reader (Molecular Devices). The plate was incubated at $37^{\circ} \mathrm{C}$ for the duration of the experiment.

\section{Statistical analysis}

Data set distributions were tested for normality using the Shapiro-Wilk test [48]. As all replicates were normally distributed, differences in means were analysed using a 1-way Analysis of Variance (ANOVA) with a 95\% confidence interval, with Tukey HSD used as a post-hoc test [49]. All statistical analyses were performed using Statistix 8 (Analyical Software, Tallahassee, FI.).

\section{Results}

Characterisation of the effects of epigenetic modulators on An. arabiensis phenotypes

\section{The effect of epigenetic modulators on oviposition and larval hatch percentage}

DZNep and genistein exposures affected adult fecundity. In SENN adults, egg production was significantly reduced when DZNep treated females were crossed with control males (2-sample t-test: $p=$ $0.03 ; t=2.38)($ Fig. $1 A)$. No other differences were observed in the SENN strain (1-way ANOVA: $p=0.99$, $\left.F_{(2 ; 17)}=0.00\right)$. SENN-DDT showed no significant differences following any of the DZNep treatments (1way ANOVA: $\left.p=0.13, F_{(2 ; 17)}=2.06\right)$ (Fig. 1B). Following genistein treatment, no significant differences in total egg production were evident in either SENN or SENN-DDT (1-way ANOVA: $p=0.72, F_{(7 ; 31)}=0.63$ ) (Fig. 1C). Following genistein treatment, a significant decrease in hatch percentage was noted when both male and female SENN were treated (1-way ANOVA: $\left.p=0.03, F_{(3 ; 15)}=4.16\right)$ (Fig. 1D). There were no changes in total egg production in either SENN or SENN-DDT strains after vinclozolin exposures (1-way ANOVA: $p=0.53, F_{(7 ; 47)}=0.88$ ). This was also true for hatch percentage in SENN (1-way ANOVA: $p=0.25$,

$\left.F_{(7 ; 47)}=0.86\right)$. In the SENN-DDT strain, hatch percentage increased significantly when both males and females were treated with vinclozolin (1-way ANOVA: $\left.p=0.04, F_{(1,11)}=5.40\right)$

The effect of genistein, vinclozolin and DZNep exposure on insecticide resistance phenotype in the insecticide resistant SENN-DDT strain

Figure 2 illustrates the effects of DZNep, genistein and vinclozolin exposure on deltamethrin, malathion, permethrin, DDT and $\lambda$-cyhalothrin resistance in SENN DDT males and females. All treatments induced significantly increased deltamethrin resistance in males (2-sample t-test: DZNep: $p=0.04 ; t=2.09$, genistein: $p<0.01 ; t=4.70$, vinclozolin: $p=0.01 ; t=2.91$ ). Malathion resistance in males was also increased following exposure to vinclozolin (2-sample t-test: $p=0.01 ; t=2.66$ ) while DZNep and genistein 
exposures left malathion resistance unchanged (2-sample t-test: DZNep: $p=0.74 ; t=0.33$, genistein: $p=$ $0.58 ; \mathrm{t}=0.56)$.

Significantly increased resistance to deltamethrin was evident in females following exposure to genistein and vinclozolin (2-sample t-tests: genistein: $p=0.03 ; t=2.22$, vinclozolin: $p=0.02 ; t=2.41$ ) with DZNep exposure leaving deltamethrin resistance unchanged (2-sample t-test: $p=0.45 ; t=-0.75)$. Malathion resistance in females was only the only phenotype affected by DZNep exposure, with a significant increase mortality (2-sample t-test: $p=0.04 ; t=-2.07$ ), while genistein and vinclozolin exposures exerted no insecticide resistance phenotypic effects (2-sample t-test: genistein: $p=0.42 ; t=-0.82$, vinclozolin: $p=0.22$; $t=1.24)$. None of the treatments affected female permethrin resistance (2-sample t-test: $D Z N e p: p=0.09$; $t=-1.77$, genistein: $p=0.84 ; t=0.14$, vinclozolin: $p=0.07 ; t=-1.9$ ). Vinclozolin exposures significantly increased DDT and $\lambda$-cyhalothrin resistance in adult females (2-sample t-test: DDT: $p=0.03 ; t=2.22, \lambda$ cyhalothrin: $p<0.01 ; t=4.47$ ) with all other exposures remaining unchanged (2-sample t-test: DDT DZNep: $p=0.30 ; t=-1.1$, DDT genistein: $p=0.47 ; t=0.73, \lambda$-cyhalothrin DZNEP: $p=0.60 ; t=-0.52, \lambda$ cyhalothrin genistein: $p=0.90 ; t=4.47$ ).

Characterisation of epigenetic markers in adult An. arabiensis emerging from larvae reared in metalpolluted water

\section{Quantification of m6A RNA methylation levels}

RNA methylation is a common modification of epigenetic architecture at the RNA level. Figure 3 illustrates the global m6A RNA methylation patterns for SENN and SENN-DDT when exposed to the heavy metal pollutants $\mathrm{CdCl}_{2}, \mathrm{Cu}\left(\mathrm{NO}_{3}\right)_{2}$ and $\mathrm{Pb}\left(\mathrm{NO}_{3}\right)_{2}$.

For male SENN adults, only lead treatments showed increased methylation patterns (2-sample t-test: $p=$ $0.02 ; t=-3.50)$ while other treatments remained unchanged (2-sample t-test: control vs cadmium: $p=0.81$; $t=0.26$, control vs copper: $p=0.42 ; t=0.88$ ). No RNA methylation patterns for female SENN changed significantly following pollutant exposure (2-sample t-test: control vs cadmium: $p=0.26 ; t=-1.30$, control vs copper: $p=0.19 ; t=1.57$, control vs lead: $p=0.57 ; t=-0.62$ ).

Lead treatments decreased methylation patterns in SENN-DDT males (2-sample t-test: $p=0.04 ; t=2.90$ ), while other treatments remained unchanged (2-sample t-test: control vs cadmium: $p=0.35 ; t=-1.05$, control vs copper: $p=0.30 ; t=1.17$ ). For SENN-DDT females, significant RNA methylation decreases were evident following copper treatments (2-sample t-test: $p=0.03 ; t=3.07$ ), with other treatments remaining unchanged (2-sample t-test: control vs cadmium: $p=0.08 ; t=2.95$, control vs lead: $p=0.72 ; t=0.38$ ).

SENN control, copper and lead treatments all showed differences between males and females (2-sample

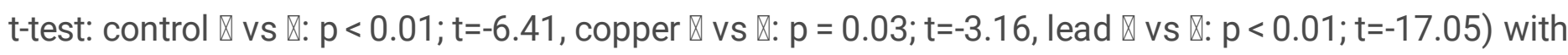
cadmium remaining unchanged (2-sample t-test: $p=0.19 ; t=-1.57$ ). SENN-DDT treatments only showed significant differences for untreated conditions (2-sample t-test: $p<0.01 ; t=4.87$ ) with treated conditions

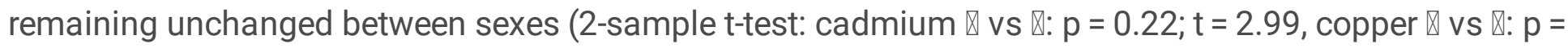

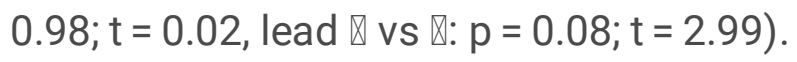


When comparing methylation levels between strains, SENN-DDT females had higher methylation under control conditions (1-way ANOVA: $\left.p<0.01, F_{(1 ; 5)}=62.1\right)$, but only again when treated with lead (1-way ANOVA: $\left.p=0.04, F_{(1 ; 5)}=7.76\right)$. When comparing males, the SENN strain had higher methylation levels (1way ANOVA: $\left.p=0.04, F_{(1 ; 5)}=9.04\right)$ as well as when treated by lead $\left(1\right.$-way ANOVA: $\left.p<0.01, F_{(1 ; 5)}=310\right)$

\section{Quantification of DNA methylation levels}

DNA methylation level can be quantified by calorimetric quantification of $5 \mathrm{hmc}$ and 5-mC residues. 5$\mathrm{hmC}$, a form of DNA modification, can provide details on the distribution of methylation patterns as it is typically concentrated in exonic regions of genomic DNA, while 5-mC is implicated in cellular growth [50].

To ascertain the potential effects of epigenetic modifiers on the mosquitoes, 5-mC and 5-hmC levels were quantified to determine any effects on gene expression (Fig. 4).

There was no significant differences in 5-mC level in SENN males after any of the treatments (1-way ANOVA $\left.p=0.07, F_{(3 ; 11)}=3.56\right)$. The same was true for SENN females $\left(1\right.$-way ANOVA $p=0.17, F_{(3 ; 11)}=$ 2.14). By contrast, there was a significant difference after genestein treatment in SENN-DDT males (1way ANOVA $\left.p=0.02, F_{(1 ; 5)}=13.5\right)$. Female SENN-DDT with all treatments resulting in an increased level of 5-mC methylation (1-way ANOVA $\left.\mathrm{p}<0.01, \mathrm{~F}_{(3 ; 11)}=9.69\right)$. There was no difference between males and females in SENN (1-way ANOVA $\left.p=0.07, F_{(7 ; 23)}=2.40\right)$. This was not so in SENN-DDT, where under untreated conditions, females had lower 5-mC levels, but this effect was lost after treatment (1-way ANOVA $p<0.01, F_{(7 ; 23)}=7.27$; Critical Q-value: 4.903; Standard error for comparison: 0.9615; Critical value for comparison: 3.3334$)$.

When examining the effect of the treatment between strains, there was no difference between SENN and SENN-DDT males (1-way ANOVA $p=0.02, F_{(7 ; 23)}=3.25$; Critical Q-value: 4.903; Standard error for comparison: 1.2217; Critical value for comparison: 4.2356). When comparing SENN and SENN-DDT females, the only difference presented under untreated conditions (1-way ANOVA $p<0.01, F_{(7 ; 23)}=4.56$; Critical Q-value: 4.903; Standard error for comparison: 1.2217; Critical value for comparison: 4.2356)

All adult SENN male treatments showed significantly lowered 5-hmC patterns compared to the untreated control males (2-sample t-test: control vs DZNEP: $p<0.01 ; t=12.59$, control vs genistein: $p<0.01 ; t=9.02$, control vs vinclozolin: $p=0.01 ; t=3.84$ ). Female SENN adults exposed to genistein and vinclozolin showed significantly increased relative methylation patterns (2-sample t-test: control vs genistein: $p<$ 0.01 ; $t=-5.58$, control vs vinclozolin: $p=0.03$; $t=-3.03$ ) (Fig. 4).

There were no significantly different 5-hmC methylation patterns in male SENN DDT samples compared to their control (1-way ANOVA: $\left.p=0.19, F_{(3,14)}=1.91\right)$. Adult females, however, showed significant increases following DZNep and vinclozolin exposures (2-sample t-test: control vs DZNEP: $p=0.01$; $t=-4.49$, control vs vinclozolin: $p=0.03 ; t=-3.22$ ) (Fig. 4). 
The potential effects of larval metal exposure on DNA methylation, expressed as relative 5-mC and 5-hmC residues (demethylation and methylation), were quantified by ELISA (Fig. 5). SENN adult males showed significantly higher concentrations of 5-mC residues than the untreated controls after all treatments (1way ANOVA: $\left.p<0.01, F_{(3,11)}=30.2\right)$. Female SENN adults also showed significantly different responses to larval metal exposure (1-way ANOVA: $p=0.02, F_{(3,11)}=6.28$ ), with lead and copper resulting in significantly lower 5 -mC levels, and cadmium significantly higher levels. Moreover, differences were evident between sexes in all SENN cases (1-way ANOVA: $\left.p<0.01, F_{(7,23)}=11.2\right)$.

SENN-DDT males showed a significant increase in 5-mC concentrations after cadmium and lead treatments and significantly lower levels after copper treatment (1-way ANOVA: $p<0.01, F_{(3,11)}=69.9$ ). SENN-DDT females showed significantly lower 5-mC levels following copper and cadmium treatments, but lead treatment induced significantly higher levels (1-way ANOVA: $p=0.02, F_{(3,11)}=5.87$ ). There were evident differences between the sexes in adult SENN treatments, but only after cadmium and lead treatments (1-way ANOVA: $\left.p<0.01, F_{(7,23)}=24.0\right)$ (Fig. 5A).

When comparing 5-mC levels between strains, only the females differed significantly between strains (1way ANOVA: $p=0.04, F_{(3,11)}=3.9$; Tukey's critical Q: 4.257 , standard error for comparison: 1.0805 , Critical value for comparison: 3.4589). Cadmium treatment did not result in a significant difference between strains (1-way ANOVA: $\left.p=0.15, F_{(3,11)}=2.38\right)$. Similarly, copper treatment did not result in a significant difference between strains (1-way ANOVA: $\left.p=0.52, F_{(3,11)}=0.80\right)$. This was also true for lead treatment (1-way ANOVA: $\left.p=0.33, F_{(3,11)}=1.33\right)$.

SENN males showed significantly lower 5-hmC levels following larval metal treatments (1-way ANOVA: $p$ $\left.<0.01, F_{(3,11)}=129\right)$ (Fig. 5B). For SENN females, only lead treatment significantly decreased 5-hmC levels, while all copper and cadmium treatments significantly increased 5-hmC levels (1-way ANOVA: $\mathrm{p}<$ $\left.0.01, F_{(3,11)}=21.6\right)$ (Fig. 5B). Significant differences between males and females were only found in the untreated controls (1-way ANOVA: $\left.p<0.01, F_{(7,23)}=103\right)$.

For SENN-DDT males, no significant differences were observed in any of the treatment groups (1-way ANOVA: $\left.p=0.31, F_{(3,11)}=1.42\right)$. Similarly, no treatments affected the 5 -hmC levels of lead treatment resulted in a significant decrease in 5-hmC levels in SENN DDT females (1-way ANOVA: $p=0.12, F_{(3,8)}=$ 3.07). Significant differences were evident between copper and lead-treated males and females (1-way ANOVA: $\left.p=0.02, F_{(7,23)}=3.56\right)$.

When comparing 5-hmC levels between strains, SENN control males had significantly higher levels, but SENN-DDT males and females, as well as SENN females did not differ significantly (1-way ANOVA: $p<$ $0.01, F_{(3,11)}=215$; Tukey's critical Q: 4.257, standard error for comparison: 0.009 , Critical value for comparison: 0.0292). Cadmium treatment did not result in a significant difference between strains (1-way ANOVA: $\left.p=0.14, F_{(3,11)}=2.47\right)$. After copper treatment, SENN males and SENN-DDT females did not differ, while SENN-DDT males and SENN females did not differ significantly (1-way ANOVA: $p=0.01$, 
$F_{(3,11)}=7.45$; Tukey's critical Q: 4.257 , standard error for comparison: 0.0159 , Critical value for comparison: 0.0508). Following lead treatment SENN and SENN-DDT females differed significantly, but males did not differ significantly (1-way ANOVA: $p=0.04, F_{(3,11)}=4.13$, Tukey's critical Q: 4.257, standard error for comparison: 0.0120, Critical value for comparison: 0.0383).

\section{Quantification of histone acetyl transferase activity}

Larval metal exposure had a marked effect on HAT activity. For SENN males, all treatments resulted in a significant decrease in HAT activity (1-way ANOVA: $\left.p<0.01, F_{(3,23)}=47.4\right)$. This was also true for SENN females (1-way ANOVA: $\left.p<0.01, F_{(3,23)}=11\right)$. By contrast, SENN DDT males showed increased HAT activity after larval metal exposure (1-way ANOVA: $\left.p<0.01, F_{(3,23)}=25.9\right)$ as did female SENN-DDT (1way ANOVA: $p<0.01, F_{(3,23)}=8.71$ ) (Fig. 6).

When comparing HAT activity between strains, there was a significant difference between SENN and SENN-DDT under control conditions. SENN had higher HAT activity levels than SENN-DDT, but there was no difference between males and females of the same strain (1-way ANOVA: $p<0.01, F_{(3,23)}=8.71$ ). Cadmium treatment did not result in a significant difference between strains (1-way ANOVA: $p=0.75, F_{(3,}$ 23) $=0.40$ ). Copper treatment resulted in significantly higher HAT activity in SENN-DDT, but there was no difference between males and females of the same strain (1-way ANOVA: $p<0.01, F_{(3,23)}=8.49$ ). This was also true for lead treatment (1-way ANOVA: $\left.p<0.01, F_{(3,23)}=37.2\right)$.

\section{Discussion}

\section{Epigenetic manipulation can alter phenotype}

There is little information on the epigenetic architecture of mosquitoes, especially in terms of links between epigenetics and phenotypic expression.

The effect of the epigenetic modulator DZNep on fertility has been examined in An. gambiae [39], while the reductive effects of genistein and vinclozolin on imidacloprid sensitivity have been described in Aedes albopictus [19]. These epigenetic modulators exerted phenotypic effects on the SENN and SENN DDT strains as well. Despite the differences in administration of DZNep (shorter exposure time than in other protocols), treatment of SENN males resulted in a significant decrease in egg numbers, although hatching was unaffected. This shows that the insecticide susceptible SENN strain is more sensitive to DZNep, which appears to primarily affect males. The reduced hatching after SENN males and females were treated with genistein is noteworthy for the same reasons as DZNep.

Although DZNep, genistein and vinclozolin did not affect the fertility of SENN-DDT, they did affect the expression of insecticide resistance in this strain. Exposure to these modulators generally decreased insecticide-induced mortality, with all modulators decreasing deltamethrin-induced mortality in males. The exception was a DZNep-induced increase in malathion mortality in females. Vinclozolin induced the 
greatest effect on insecticide resistance in males and females. These data suggest that the epigenetic modulators may be susceptible to generalised enhanced metabolism of xenobiotics such as insecticides.

\section{Epigenetic modulators produce a change in 5-mC and 5-hmC methylation patterns}

DNA methylation can by subdivided into 5-mC and 5-hmC modifications, each of which have various implicated roles. 5-mC is implicated in DNA methylation while 5-hmC is implicated in demethylation and gene activation amongst other functions [50,51]. Of course, only some instances of increased methylation decrease gene expression and vice versa [52], as the change in gene expression is highly dependent on the methylation taking place at particular genomic regions (promoters versus exons versus silencers etc.). This therefore makes accurately predicting changes in gene expression by methylation versus demethylation on a global scale very difficult without more sensitive techniques.

DNA methylation, as assessed by $5-\mathrm{mC}$ methylation levels, was affected by dietary supplementation with the epigenetic modulators genestein, vinclozolin, and DZNep. The 5-mC levels in the SENN strain were unaffected by the modulators. In contrast to the SENN strain, all the modulators increased 5-mC levels in SENN-DDT females, but only genestein increased levels in SENN-DDT males. It is worth noting that this pattern is opposite to the5-hmC levels, where the modulators induced changes in SENN, but not SENNDDT.

Dietary supplementation with the three epigenetic modulators did alter DNA demethylation levels. This may underlie some of the observations in the phenotypic studies. SENN was more sensitive to epigenetic modulation than SENN DDT, although all three modulators caused a significant change in 5-hmC methylation in at least one of the sexes, with genestein altering both male and female methylation. Where there were changes in the SENN strain, the modulators increased methylation in females, while decreasing it males. This combination (genestein treated male and female) evidently induced a significantly reduced larval hatch percentage. By contrast, genestein had no effect on 5-hmC methylation in SENN-DDT at all, unlike DZNep and vinclozolin exposures which were associated with, significant increases in female 5-hmC methylation. The increase in vinclozolin-induced 5-hmC methylation may be linked to the marked decrease in female DDT and pyrethroid resistance observed after treatment.

\section{Larval heavy metal exposure alters the epigenetic profile of adult mosquitoes}

The precise role of RNA methylation in epigenetic regulation is poorly understood. It is believed to be associated with physical changes in the messenger RNA (mRNA) structure, namely $\mathrm{N}^{6}$-methyl adenosine (m6A) addition. Such additions occur on both mRNA and long non-coding RNA transcripts and have implications in mRNA splicing, cellular transport, stability and immune tolerance [53]. mRNA modification is therefore proposed to act as an epigenetic marker and may potentially allow for mediation by DNA and histone modification mechanisms [54]. It is possible that because RNA methylation effects may be seen in downstream epigenetic modifications, this is the epigenetic architecture that is least affected by metals. What is noteworthy are the significant differences between males and females in SENN, where with the exception of treatment with cadmium, males always had significantly higher levels of m6A 
mRNA methylation. mRNA methylation is the only type of methylation where metal treatment resulted in more changes in SENN-DDT than in SENN, and while lead nitrate induced an increase in RNA methylation in SENN, copper nitrate treatment reduced methylation in SENN-DDT males and lead nitrate reduced methylation in males. Metal treatments therefore did not alter RNA methylation levels to a great extent.

Nucleic acid methylation varies greatly in invertebrates, and this is also true for mosquitoes. Although Aedes aegypti has been reported to be unmethylated [55], 5-mC methylation patterns were well detected in both strains of An. arabiensis. This supports previous detection of 5-mC detection in An. gambiae [7], which showed detectable levels of this methylation type using blotting techniques. In the SENN strain, 5$\mathrm{mC}$ methylation levels always differed between males and females, with control and cadmium chloride treated females being higher, while copper and lead nitrate treated males showing higher levels of 5-mC. For both SENN and SENN-DDT, all treatments resulted in significant changes in 5-mC methylation in males. In SENN males, all treatments increased methylation, while only cadmium chloride treatment increased methylation with lead and copper nitrate exposure resulting in a significant reduction in 5-mC methylation. This further suggests that SENN is particularly susceptible to heavy metal-induced change on an epigenetic level and that exposure to such pollutants heavily alters the DNA epigenetic architecture of this strain.

The 5-hmC methylation patterns observed after modulator exposure were congruent with the patterns observed after larval metal exposure, where SENN-DDT was largely unaffected by metal treatment, except after copper nitrate treatment where female SENN-DDT 5-hmC methylation was higher than that of the males. All treatments reduced methylation in SENN males, but only lead nitrate induced a significant decrease in females.

As 5-mC and 5-hmC methylation are generally associated with methylation and demethylation, respectively, and, therefore, broad silencing and expression [50,51], their levels could roughly be the inverse of each other within the same individual. In these data, there was a generalised pattern of lower 5$\mathrm{hmC}$ patterns where the $5-\mathrm{mC}$ was high, suggesting that this methylation-silencing/demethylationactivation pattern may also be a regulatory mechanism in An. arabiensis.

HAT activity reveals the most marked pattern changes due to metal exposure. There was a clear suppression of HAT activity with metal treatment in both males and females of the SENN strain. This pattern was inverted in SENN-DDT, with metals increasing HAT activity. Lead nitrate exposure resulted in the lowest HAT activity for SENN, but the highest for SENN-DDT.

\section{Significance of changes in epigenetic architecture}

A key discovery of this study on a basal level was that both SENN and SENN-DDT showed sex differences in methylation at the RNA, but not the DNA level. This may have implications for regulation of sex-specific behaviours such as blood feeding. There may therefore be numerous sex-specific differences in An. arabiensis that have an epigenetic basis. 
The marked differences in methylation patterns in SENN and SENN-DDT support the work of Oppold et al [19] who demonstrated an epigenetic element to insecticide resistance in Aedes albopictus. The insecticide susceptible SENN strain is also far more sensitive to alteration in epigenetic signatures after pollutant stress. This may be the underlying reason why metal pollution results in more rapid transgenerational selection for insecticide tolerance than the SENN-DDT strain [38].

5-mC methylation is associated with gene silencing. In the SENN strain, this methylation pattern was generally increased after larval metal exposure. 5 -hmC methylation, associated with gene activation, was generally decreased in this strain. In addition to reduced detoxification enzyme activity in SENN, this may explain the toxicity of first generation metal exposure in this strain as the general shift in methylation signatures point to reduced transcription. Conversely, $5-\mathrm{mC}$ methylation is decreased in the insecticideresistant SENN-DDT strain, but without a concomitant increase in 5-hmC methylation. This suggests that the epigenetic mechanism for metal tolerance may be reduced gene silencing rather than a de novo drive to gene expression. HAT activity partially supports this hypothesis, with larval metal exposure reducing HAT activity in SENN and increasing it in SENN-DDT. It is worth mentioning that the patterns of HAT activity reduction are inverse to the toxicity of the metals in $\operatorname{SENN}[29,45]$, i.e. the less toxic the metal, the more exposure to it suppresses HAT activity, which is not congruent with the defence against toxicity hypothesis suggested above. Studies of An. gambiae demonstrated that the presence of the H3K27ac modification, a mark of acetylation on Histone $\mathrm{H} 3$, is associated with active transcription [15]. This suggests that the HAT activity observed in this study may be associated with increased transcription.

The exact mechanism of histone modification is contentious. It is generally accepted that histone modification plays a more important role in recruiting non-histone proteins which bind to the areas surrounding these modifications and allow for dynamic changes in chromatin structure [56]. This may explain the lack of changes in methylation patterns in SENN-DDT, suggesting that the metal stress induces a distinctly different epigenetic mechanism in the insecticide resistant strain than the insecticide resistant strain.

\section{Conclusion}

Although global detection of epigenetic signatures is not the most sensitive mechanism of epigenetic analysis, this study provides a proof-of-concept that metal pollution has such an effect that it can be detected by ELISA techniques. Future studies could examine tissue specific or even single cell epigenetic changes. However, this study presents one of the first examination of epigenetics in the Anopheles gambiae complex. Insecticide resistant and susceptible An. arabiensis strains respond differently to epigenetic modulators, with the insecticide susceptible SENN strain being more sensitive to epigenetic alteration. Similarly, larval metal exposure induced more epigenetic alterations in SENN adults. 5-mC and 5-hmC methylation patterns were generally inverted, suggesting a relationship between methylation and demethylation. The DNA methylation patterns in SENN suggest a general decrease in gene activity, while that of SENN-DDT suggests increased gene activity after larval metal exposure, modulated by decreased 
methylation and increased HAT activity. There was therefore a marked effect of insecticide resistance on the epigenetic response of $A n$. arabiensis to metal pollution stress.

\section{Declarations}

\section{Ethics approval and consent to participate}

This project was performed under the animal ethics waiver S Oliver 03-01-2018 from the University of the Witwatersrand. No consent for participation was required as all work was performed on mosquitoes.

\section{Consent for publication}

Not applicable

\section{Availability of data and material}

All data arising from this study is available within this article

\section{Competing interests}

The authors declare no competing interests

\section{Funding}

This project was funded by the National Research Foundation of South Africa Thuthuka post-PhD programme (TTK160622173523), as well as the Competitive Support for Unrated Researchers programme (SRUG190313423259) and a development grant from the National Health Laboratory Service (2014-DEV-SOL1).

\section{Authors' contributions}

ACSNJ and SVO performed the experiments, analysed the data and produced the initial drafts of the manuscript. BDB and SVO produced the final manuscript.

\section{Acknowledgements}

Dr Rodney Hull is thanked for his constructive comments on the manuscript.

\section{References}

1. Elgin SC, Reuter G: Position-effect variegation, heterochromatin formation, and gene silencing in Drosophila.Cold Spring Harb Perspect Biol 2013, 5:a017780.

2. Bonasio R, Li Q, Lian J, Mutti NS, Jin L, Zhao H, Zhang P, Wen P, Xiang H, Ding Y, et al: Genome-wide and caste-specific DNA methylomes of the ants Camponotus floridanus and Harpegnathos saltator.Curr Biol 2012, 22:1755-1764. 
3. Feng S, Cokus SJ, Zhang X, Chen PY, Bostick M, Goll MG, Hetzel J, Jain J, Strauss SH, Halpern ME, et al: Conservation and divergence of methylation patterning in plants and animals.Proc Natl Acad Sci U S A 2010, 107:8689-8694.

4. Lyko F, Foret S, Kucharski R, Wolf S, Falckenhayn C, Maleszka R: The honey bee epigenomes: differential methylation of brain DNA in queens and workers.PLOS Biol 2010, 8:e1000506.

5. Bewick AJ, Vogel KJ, Moore AJ, Schmitz RJ: Evolution of DNA Methylation across Insects.Mol Biol Evol 2017, 34:654-665.

6. Misof B, Liu S, Meusemann K, Peters RS, Donath A, Mayer C, Frandsen PB, Ware J, Flouri T, Beutel RG, et al: Phylogenomics resolves the timing and pattern of insect evolution.Science 2014, 346:763767.

7. Marhold J, Rothe N, Pauli A, Mund C, Kuehle K, Brueckner B, Lyko F: Conservation of DNA methylation in dipteran insects. Insect Mol Biol 2004, 13:117-123.

8. Sharakhov IV, Sharakhova MV: Heterochromatin, histone modifications, and nuclear architecture in disease vectors.Curr Opin Insect Sci 2015, 10:110-117.

9. Cedar H, Bergman Y: Linking DNA methylation and histone modification: patterns and paradigms.Nat Rev Genet 2009, 10:295-304.

10. Glastad KM, Hunt BG, Goodisman MA: DNA methylation and chromatin organization in insects: insights from the Ant Camponotus floridanus.Genome Biol Evol 2015, 7:931-942.

11. Glastad KM, Hunt BG, Goodisman MAD: Epigenetics in Insects: Genome Regulation and the Generation of Phenotypic Diversity.Annu Rev Entomol 2019, 64:185-203.

12. Suganuma T, Workman JL: Signals and combinatorial functions of histone modifications.Annu Rev Biochem 2011, 80:473-499.

13. Kharchenko PV, Alekseyenko AA, Schwartz YB, Minoda A, Riddle NC, Ernst J, Sabo PJ, Larschan E, Gorchakov AA, Gu T, et al: Comprehensive analysis of the chromatin landscape in Drosophila melanogaster.Nature 2011, 471:480-485.

14. Negre N, Brown CD, Ma L, Bristow CA, Miller SW, Wagner U, Kheradpour P, Eaton ML, Loriaux P, Sealfon R, et al: A cis-regulatory map of the Drosophila genome.Nature 2011, 471:527-531.

15. Gomez-Diaz E, Rivero A, Chandre F, Corces VG: Insights into the epigenomic landscape of the human malaria vector Anopheles gambiae.Front Genet 2014, 5:277.

16. Kubota T: Epigenetic alterations induced by environmental stress associated with metabolic and neurodevelopmental disorders. Environ Epigenet 2016, 2:dvw017.

17. Lamke J, Baurle I: Epigenetic and chromatin-based mechanisms in environmental stress adaptation and stress memory in plants.Genome Biol 2017, 18:124.

18. Kress A, Oppold AM, Kuch U, Oehlmann J, Muller R: Cold tolerance of the Asian tiger mosquito Aedes albopictus and its response to epigenetic alterations.J Insect Physiol 2017, 99:113-121.

19. Oppold A, Kress A, Vanden Bussche J, Diogo JB, Kuch U, Oehlmann J, Vandegehuchte MB, Muller R: Epigenetic alterations and decreasing insecticide sensitivity of the Asian tiger mosquito Aedes 
albopictus.Ecotoxicol Environ Saf 2015, 122:45-53.

20. Sinka ME, Bangs MJ, Manguin S, Coetzee M, Mbogo CM, Hemingway J, Patil AP, Temperley WH, Gething PW, Kabaria CW, et al: The dominant Anopheles vectors of human malaria in Africa, Europe and the Middle East: occurrence data, distribution maps and bionomic precis.Parasit Vectors 2010, 3:117.

21. Dandalo LC, Brooke BD, Munhenga G, Lobb LN, Zikhali J, Ngxongo SP, Zikhali PM, Msimang S, Wood OR, Mofokeng M, et al: Population Dynamics and Plasmodium falciparum (Haemosporida: Plasmodiidae) Infectivity Rates for the Malaria Vector Anopheles arabiensis (Diptera: Culicidae) at Mamfene, KwaZulu-Natal, South Africa.J Med Entomol 2017, 54:1758-1766.

22. Kitau J, Oxborough RM, Tungu PK, Matowo J, Malima RC, Magesa SM, Bruce J, Mosha FW, Rowland MW: Species shifts in the Anopheles gambiae complex: do LLINs successfully control Anopheles arabiensis? PLoS One 2012, 7:e31481.

23. Killeen GF: Characterizing, controlling and eliminating residual malaria transmission.Malar J 2014, 13:330.

24. Knox TB, Juma EO, Ochomo EO, Pates Jamet H, Ndungo L, Chege P, Bayoh NM, N'Guessan R, Christian RN, Hunt RH, Coetzee M: An online tool for mapping insecticide resistance in major Anopheles vectors of human malaria parasites and review of resistance status for the Afrotropical region.Parasit Vectors 2014, 7:76.

25. Azrag RS, Mohammed BH: Anopheles arabiensis in Sudan: a noticeable tolerance to urban polluted larval habitats associated with resistance to Temephos.Malar J 2018, 17:204.

26. Jones CM, Toe HK, Sanou A, Namountougou M, Hughes A, Diabate A, Dabire R, Simard F, Ranson H: Additional selection for insecticide resistance in urban malaria vectors: DDT resistance in Anopheles arabiensis from Bobo-Dioulasso, Burkina Faso.PLoS One 2012, 7:e45995.

27. Gillies MT, Meillon BD: The Anophelinae of Africa south of the Sahara (Ethiopian Zoogeographical Region).Publications of the South African Institute for Medical Research 1968, 54.

28. Kamdem C, Fouet C, Gamez S, White BJ: Pollutants and Insecticides Drive Local Adaptation in African Malaria Mosquitoes.Mol Biol Evol 2017, 34:1261-1275.

29. Oliver SV, Brooke BD: The effect of metal pollution on the life history and insecticide resistance phenotype of the major malaria vector Anopheles arabiensis (Diptera: Culicidae).PLoS One 2018, 13:e0192551.

30. Poupardin R, Reynaud S, Strode C, Ranson H, Vontas J, David JP: Cross-induction of detoxification genes by environmental xenobiotics and insecticides in the mosquito Aedes aegypti: impact on larval tolerance to chemical insecticides.Insect Biochem Mol Biol 2008, 38:540-551.

31. Jeanrenaud ACSN, Brooke BD, Oliver SV: The effects of larval organic fertiliser exposure on the larval development, adult longevity and insecticide tolerance of zoophilic members of the Anopheles gambiae complex (Diptera: Culicidae).PLoS One 2019, 14:e0215552.

32. Oliver SV, Brooke BD: The effect of commercial herbicide exposure on the life history and insecticide resistance phenotypes of the major malaria vector Anopheles arabiensis (Diptera: culicidae).Acta 
Trop 2018, 188:152-160.

33. Oliver SV, Brooke BD: The effect of multiple blood-feeding on the longevity and insecticide resistant phenotype in the major malaria vector Anopheles arabiensis (Diptera: Culicidae).Parasit Vectors 2014, 7:390.

34. Oliver SV, Brooke BD: The effect of elevated temperatures on the life history and insecticide resistance phenotype of the major malaria vector Anopheles arabiensis (Diptera: Culicidae).Malar J 2017, 16:73.

35. Oliver SV, Brooke BD: The effect of larval nutritional deprivation on the life history and DDT resistance phenotype in laboratory strains of the malaria vector Anopheles arabiensis.Malar J 2013, 12:44.

36. Venter N, Oliver SV, Muleba M, Davies C, Hunt RH, Koekemoer LL, Coetzee M, Brooke BD: Benchmarking insecticide resistance intensity bioassays for Anopheles malaria vector species against resistance phenotypes of known epidemiological significance.Parasit Vectors 2017, 10:198.

37. Hunt RH, Brooke BD, Pillay C, Koekemoer LL, Coetzee M: Laboratory selection for and characteristics of pyrethroid resistance in the malaria vector Anopheles funestus.Med Vet Entomol 2005, 19:271275.

38. Jeanrenaud ACSN, Brooke BD, Oliver SV: Second generation effects of larval metal pollutant exposure on reproduction, longevity and insecticide tolerance in the major malaria vector Anopheles arabiensis (Diptera: Culicidae). Parasit Vectors 2020, 13:4.

39. Sharma A, Anderson TD, Sharakhov IV: Toxicological assays for testing effects of an epigenetic drug on development, fecundity and survivorship of malaria mosquitoes.J Vis Exp 2015:52041.

40. Miranda TB, Cortez CC, Yoo CB, Liang G, Abe M, Kelly TK, Marquez VE, Jones PA: DZNep is a global histone methylation inhibitor that reactivates developmental genes not silenced by DNA methylation.Mol Cancer Ther 2009, 8:1579-1588.

41. Sundaram MK, Ansari MZ, Al Mutery A, Ashraf M, Nasab R, Rai S, Rais N, Hussain A: Genistein Induces Alterations of Epigenetic Modulatory Signatures in Human Cervical Cancer Cells.Anticancer Agents Med Chem 2018, 18:412-421.

42. Nilsson E, King SE, McBirney M, Kubsad D, Pappalardo M, Beck D, Sadler-Riggleman I, Skinner MK: Vinclozolin induced epigenetic transgenerational inheritance of pathologies and sperm epimutation biomarkers for specific diseases.PLoS One 2018, 13:e0202662.

43. WHO: Test procedures for insecticide resistance monitoring in malaria vector mosquitoes.http://wwwwhoint/iris/handle/10665/2506772016.

44. Itai T, Otsuka M, Asante KA, Muto M, Opoku-Ankomah Y, Ansa-Asare OD, Tanabe S: Variation and distribution of metals and metalloids in soil/ash mixtures from Agbogbloshie e-waste recycling site in Accra, Ghana.Sci Total Environ 2014, 470-471:707-716.

45. Mireji PO, Keating J, Hassanali A, Mbogo CM, Muturi MN, Githure JI, Beier JC: Biological cost of tolerance to heavy metals in the mosquito Anopheles gambiae.Med Vet Entomol 2010, 24:101-107. 
46. Nkya TE, Akhouayri I, Kisinza W, David JP: Impact of environment on mosquito response to pyrethroid insecticides: facts, evidences and prospects.Insect Biochem Mol Biol2013, 43:407-416.

47. Bradford MM: A rapid and sensitive method for the quantitation of microgram quantities of protein utilizing the principle of protein-dye binding.Anal Biochem 1976, 72:248-254.

48. Shapiro SS, Wilk MB: An Analysis of Variance Test for Normality (Complete Samples).Biometrika 1965, 52:591-611.

49. Tukey JW: Comparing Individual Means in the Analysis of Variance.Biometrics 1949, 5:99-114.

50. Pfeifer GP, Kadam S, Jin SG: 5-hydroxymethylcytosine and its potential roles in development and cancer.Epigenetics Chromatin 2013, 6:10.

51. Bhutani N, Burns DM, Blau HM: DNA demethylation dynamics.Cel/2011, 146:866-872.

52. Hamilton JP: Epigenetics: principles and practice.Dig Dis 2011, 29:130-135.

53. Kariko K, Buckstein M, Ni H, Weissman D: Suppression of RNA recognition by Toll-like receptors: the impact of nucleoside modification and the evolutionary origin of RNA.Immunity 2005, 23:165-175.

54. Liu N, Pan T: RNA epigenetics.Trans/ Res 2015, 165:28-35.

55. Falckenhayn C, Carneiro VC, de Mendonca Amarante A, Schmid K, Hanna K, Kang S, Helm M, Dimopoulos G, Fantappie MR, Lyko F: Comprehensive DNA methylation analysis of the Aedes aegypti genome.Sci Rep 2016, 6:36444.

56. Peterson CL, Laniel MA: Histones and histone modifications.Curr Bio/ 2004, 14:R546-551.

\section{Figures}



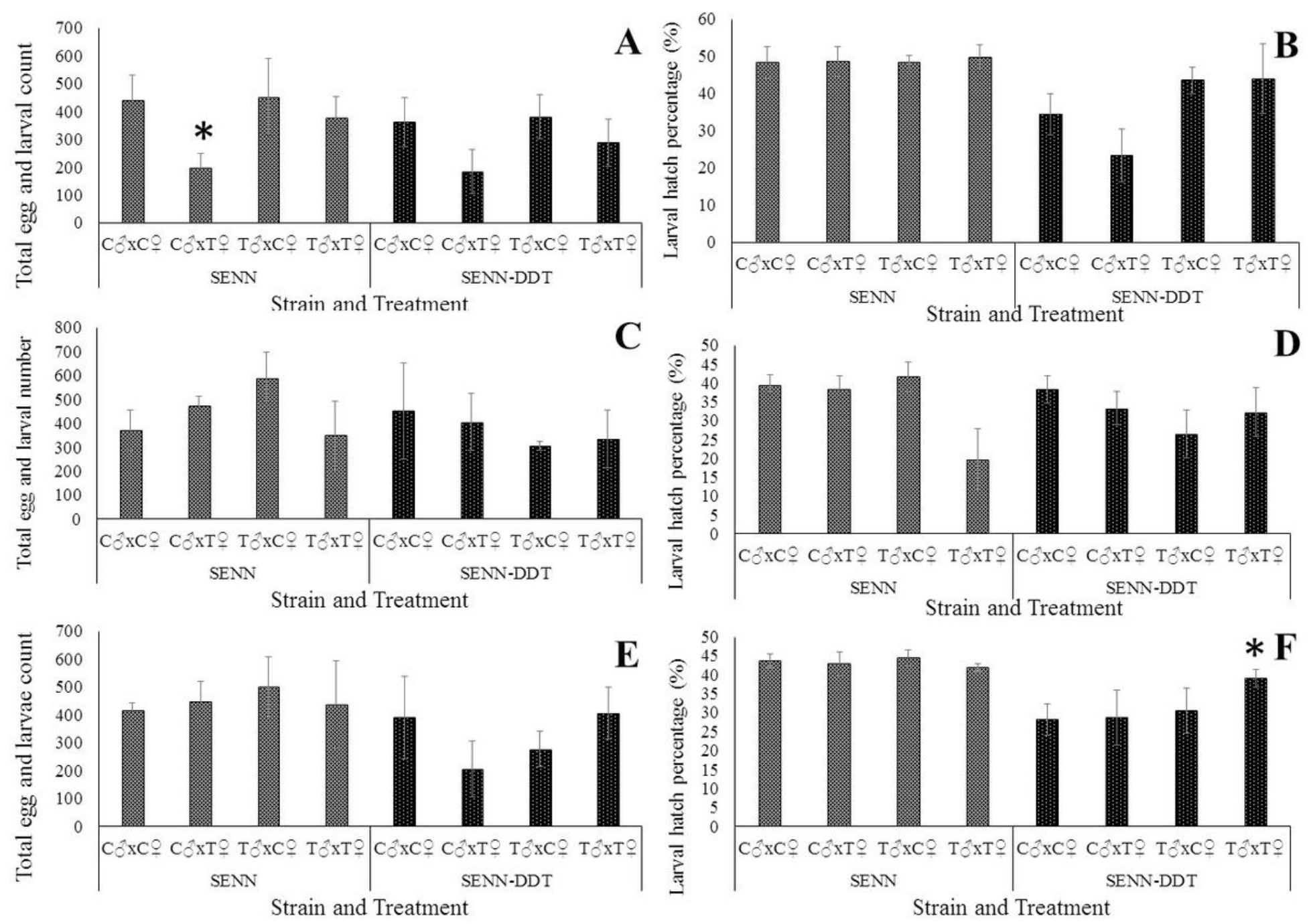

Figure 1

The effect of adult exposure to epigenetic modifier-supplemented sugar water on total number of eggs laid and hatch percentage in the An. arabiensis strains SENN and SENN- DDT. A: Total egg count after DZNep exposure. B: Hatch percentage after DZNep exposure. C: Total egg count after genistein exposure D Hatch percentage after genistein exposure. E: Total egg count after vinclozolin exposure. F: Hatch percentage after vinclozolin exposure. Asterisks $\left({ }^{*}\right)$ indicate a significant difference from the control of the same strain. 


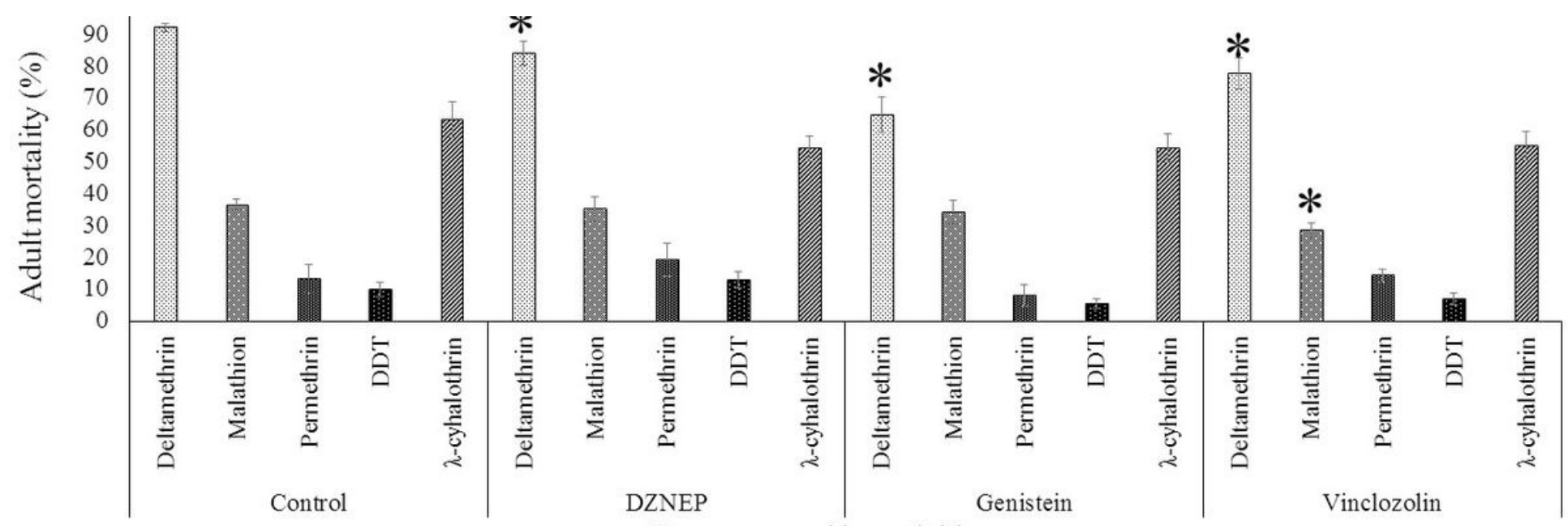

Treatment and insecticide type

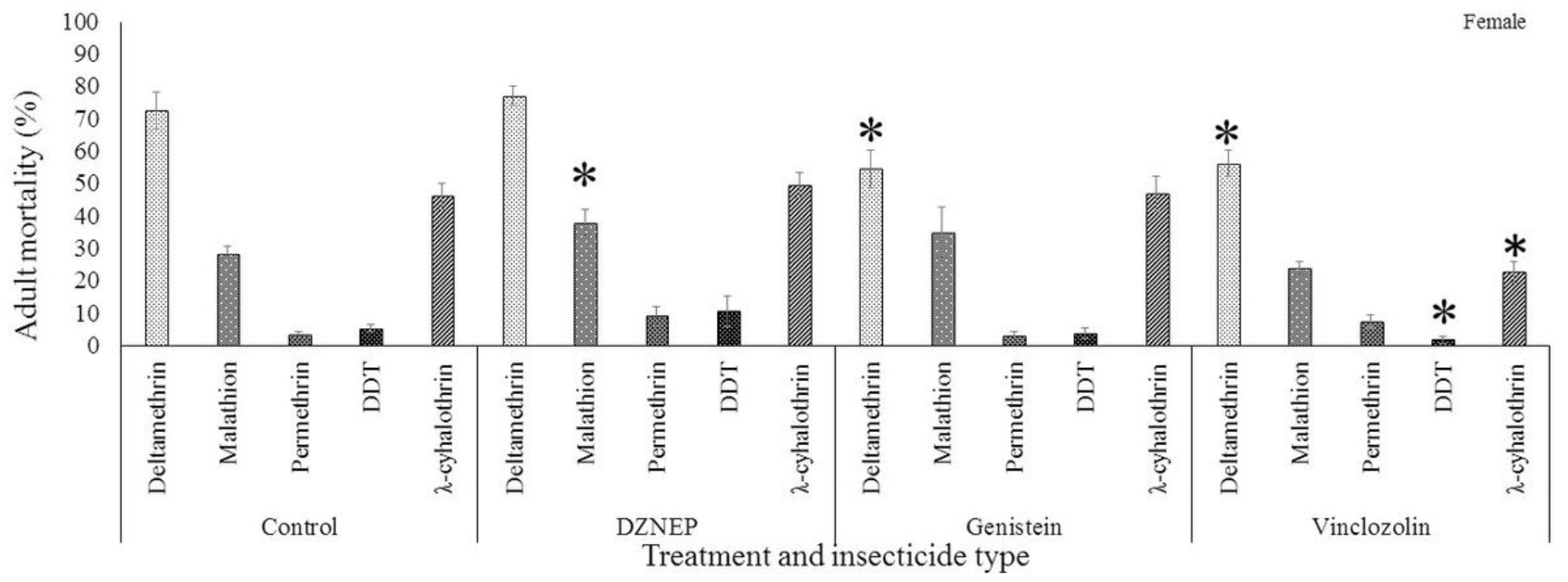

Figure 2

The effect of adult exposure to epigenetic modifier-supplemented sugar water on the insecticide resistance phenotype of the resistant An. arabiensis strain SENN- DDT. Male and female SENN-DDT mortality 24 hours post exposure to insecticides by WHO bioassay after 3 days with ad libitum access to sugar water supplemented with either DZNep, genistein or vinclozolin. Asterisks (*) indicate a significant difference from the control of the same strain. 


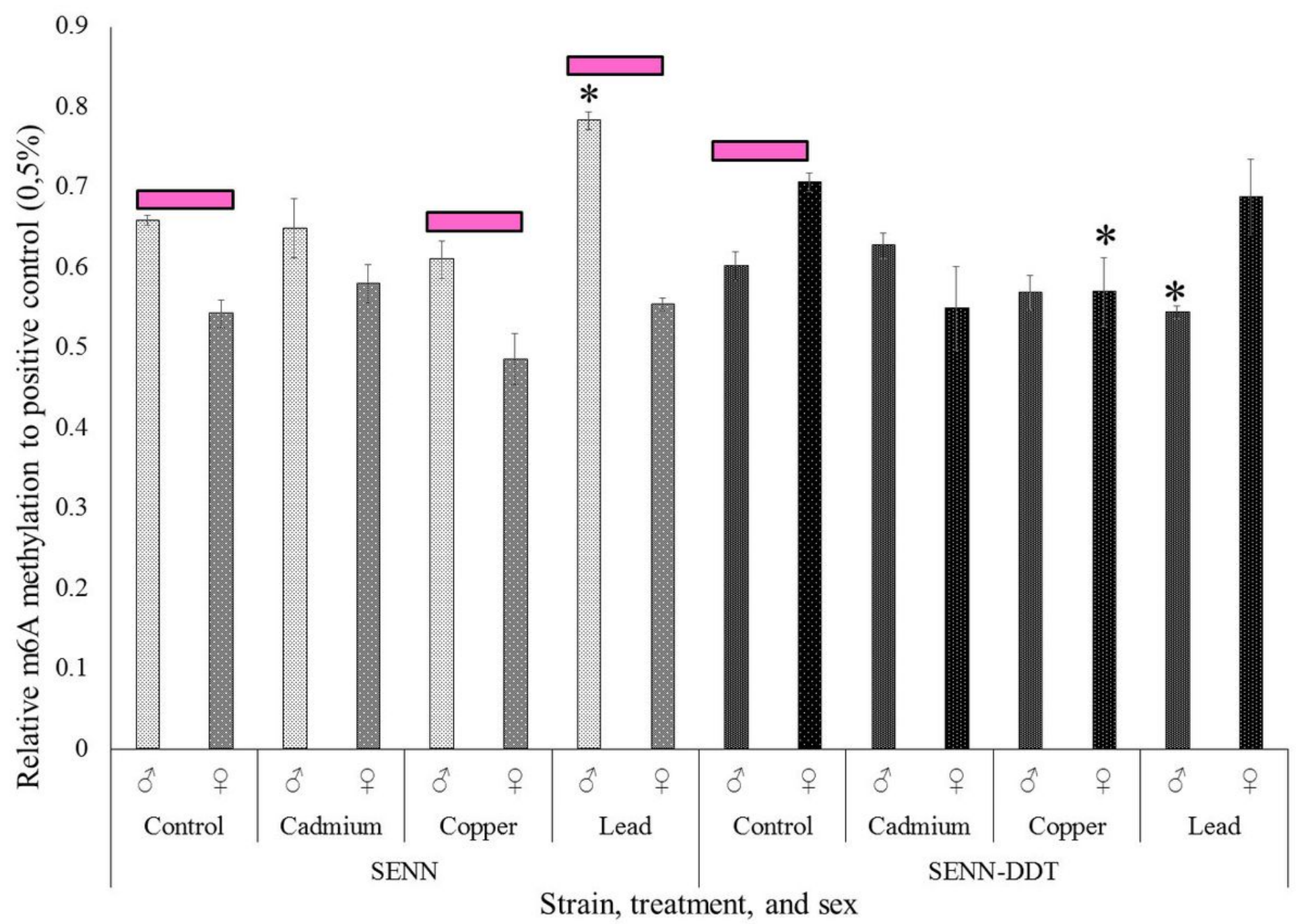

Figure 3

The effect of larval metal pollution exposure on adult RNA methylation levels in the An. arabiensis strains SENN and SENN- DDT. Asterisks $\left(^{*}\right)$ indicate a significant difference from the control of the same strain. Pink bars indicate a significant difference between males and females of the same strain and treatment. 


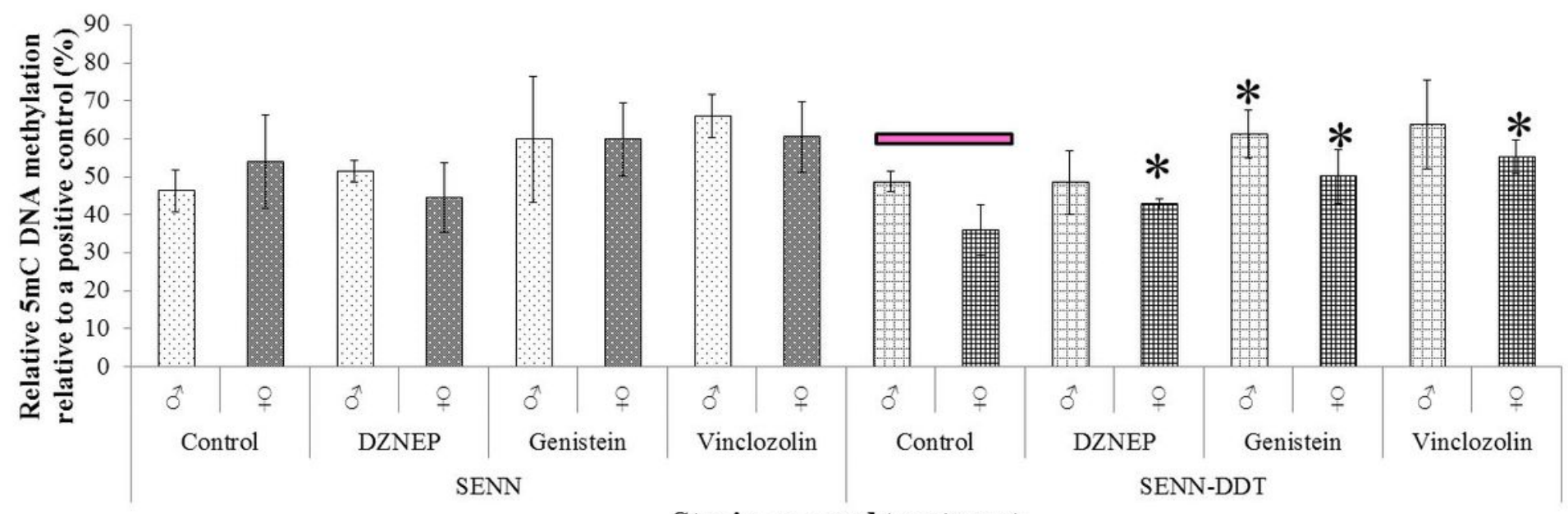

Strain, sex and treatment

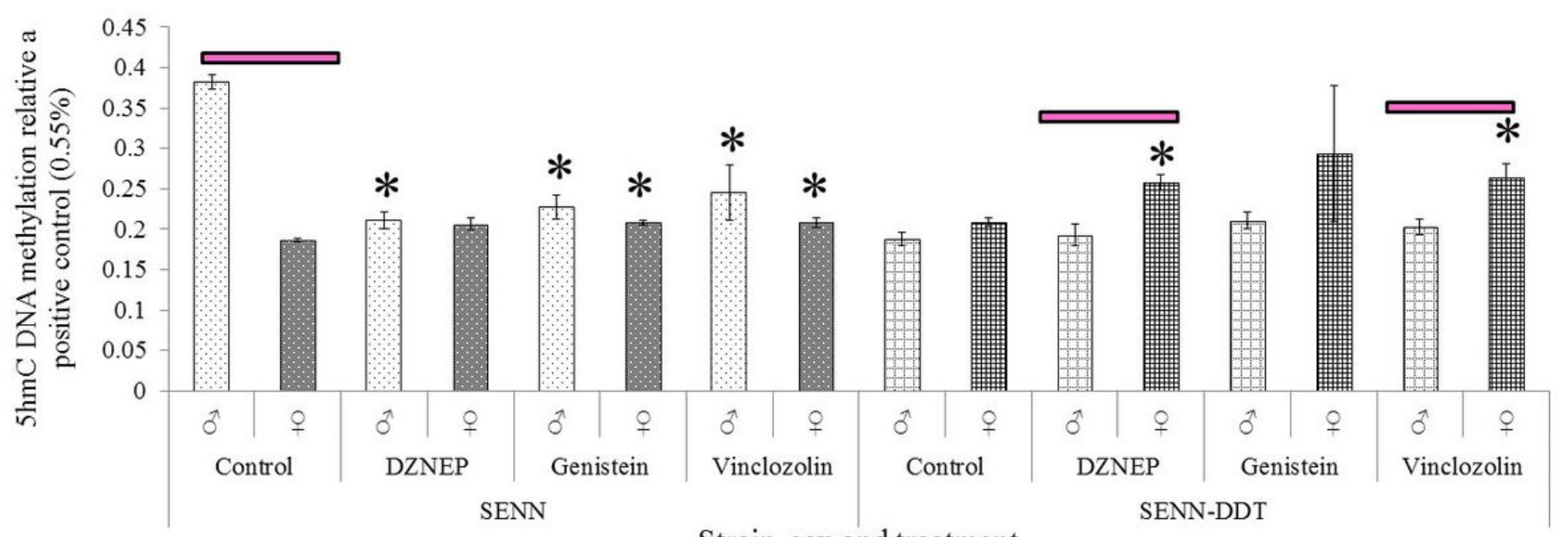

Strain, sex and treatment

Figure 4

The effect of epigenetic modifiers on adult 5-mC and 5-hmC DNA methylation levels in the An. arabiensis strains SENN and SENN- DDT. Newly emerged adults were provided with $5 \mu \mathrm{M}$ of a single modifier for 3 days. A: 5-mC methylation levels in SENN and SENN-DDT. B: 5-hmC methylation levels in SENN and SENN-DDT. Pink bars indicate a significant different between males and females. Asterisks indicate a significant difference between treatment and control of the same strain. 

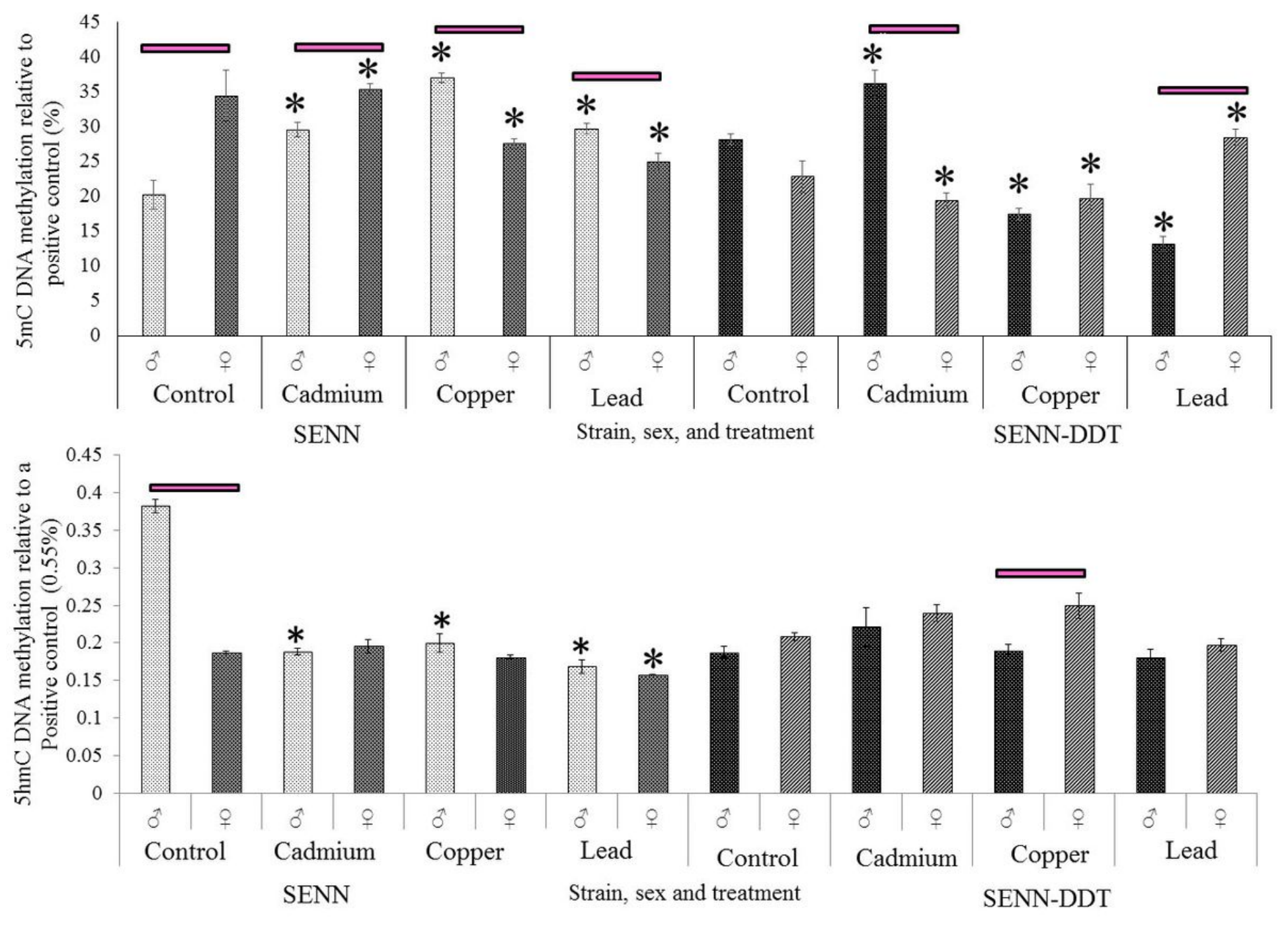

Figure 5

The effect of larval metal pollution exposure on adult DNA methylation levels in the An. arabiensis strains SENN and SENN- DDT. A: Quantification of relative 5-mC levels in adults that emerged from larvae reared in metal polluted water. B: Quantification of relative 5 -hmC levels in adults that emerged from larvae reared in metal polluted water. Pink bars indicate a significant difference between males and females. Asterisks indicate a significant difference between treatment and control of the same strain. 


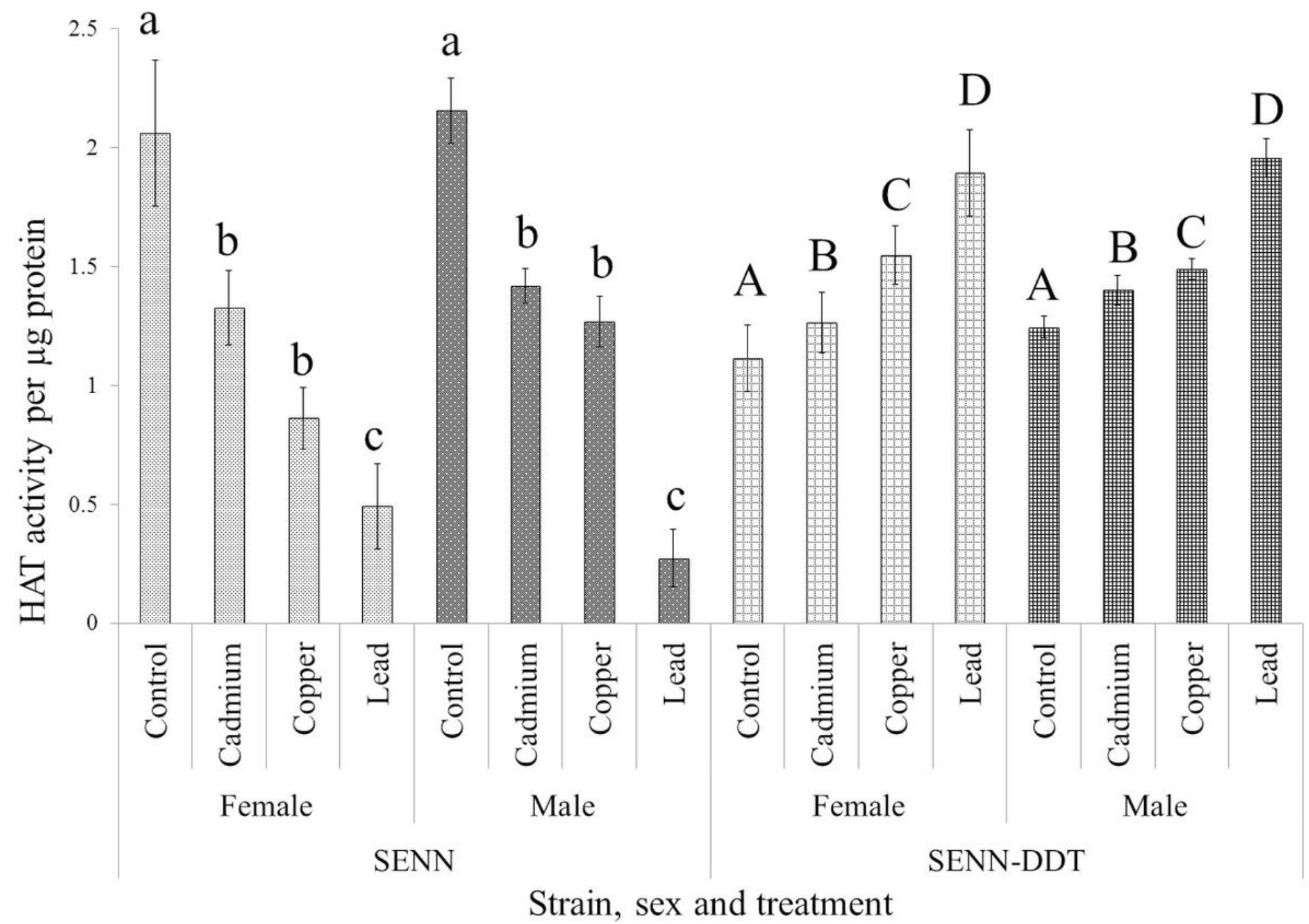

Figure 6

The effect of larval metal pollution exposure on adult Histone Acetyltransferase (HAT) activity in the An. arabiensis strains SENN and SENN- DDT. Different lower case letters indicate significant differences within the insecticide susceptible SENN strain, while different upper case letters indicate significant differences in the insecticide resistant SENN-DDT strain. 